\title{
Dynamic Data Driven Methodologies for Multiphysics System Modeling and Simulation
}

\author{
J. Michopoulos ${ }^{1}$, C. Farhat ${ }^{2}$, E. Houstis ${ }^{3,4}$, P. Tsompanopoulou ${ }^{4}$, \\ H. Zhang ${ }^{3}$, and T. Gullaud ${ }^{2}$ \\ 1 Code 6390, Special Projects Group, U.S. Naval Research Laboratory, U.S.A \\ john.michopoulos@nrl.navy.mil \\ 2 Dept. of Mechanical Engineering, Stanford University, U.S.A \\ \{cfarhat, tgullaud\}@stanford.edu \\ 3 Computer Sciences Dept. \& Electrical and Computer Engineering Dept., Purdue \\ University, U.S.A \\ \{enh, hzhang\}@purdue .edu \\ 4 Dept. of Comp. Eng. and Telecommunications, University of Thessaly, Greece
} \{enh, yota\}@uth.gr

\begin{abstract}
We are presenting a progress overview associated with our work on a data-driven environment for multiphysics applications (DDEMA). In this paper, we emphasize the dynamic-data-driven adaptive modeling and simulation aspects. Adaptive simulation examples of sensororiginating data-driven precomputed solution synthesis are given for two applications. Finally, some of the computational implementation details are presented.
\end{abstract}

\section{Introduction}

Our project has been focusing on developing methodologies and corresponding technologies, for utilizing dynamic data to generate adaptive behavioral models of physical systems as well as facilitate their realistic and near real time behavior simulation. Of particular interest here are systems that are excited by multi-field conditions and often require multi-domain modeling from a continuum systems perspective. Characteristic examples of such systems are fire dynamics simulation as well as material softening trends as a function of flight conditions of a supersonic aircraft for prediction of future behavior as detected by sensor originating data-streams.

This paper presents an overview of some of the recent progress associated with our project on adaptive modeling and simulation for supersonic aircraft response prediction for structural design, material qualification/certification needs, and fire dynamics propagation applications relevant to crisis management and decision support for first responders. It also describes the predominant features of the computational implementations. Previous progress on the project can be found elsewhere [1, 2, 3. 


\section{Adaptive Constitutive Modeling of Composites}

The concept of "data-driven system modeling" has been frequently identified by the majority of research community as the process of system identification. Various techniques exist to utilize data representing discrete values of systemic behavioral parameters (inputs/outputs) as they occur from exercising the physical system (i.e. through systematic experimentation or real time monitoring) to determine free coefficients/constants involved in the analytical (i.e. mathematical) or/and computational model of the physical system at hand. Design optimization involving objective function minimization is arguably the most popular among these techniques.

The concept of "dynamic-data-driven modeling" is an extension of the "datadriven modeling" in that it attempts to identify a system that may or may not be changing itself at real-time. In this case, the model is being formed or determined just prior to being used based on the dynamic data.

In the context of the systems associated with DDEMA we are interested in adaptively and dynamically constructing the appropriate models. We have identified two general meanings behind the term "adaptive modeling" as it has been used by various investigators. The most frequent one refers to selecting a fixed analytical representation for the model and then use the incoming dynamic data to determine the free coefficients and/or parameters associated with it. It is anticipated that as more and more data are coming in the model's identification process the model itself is adapting through the re-computation of the unknown coefficients/parameters till the data are exhausted or the model reaches its fixed point (if it exists). We call this type of adaptive modeling the "static in-line modeling" (SILM) type. However, there is a possibility that the system changes its behavior very drastically and the SILM approach does not reach fixed point nor it can reproduce older data but only recent data. This is the case for strongly non-linear systems that can be approximated by various models that can be captured by either physics aware or physics-agnostic representations [7]. This case calls for the existence of a meta-level modeling infrastructure that can select representations adapted to the data and will be designated as "dynamic meta modeling" (DMM). Clearly for each area of applicability DMM selects a model that still has to be identified by SILM. DMM is not a widely known approach especially because its computational and implementation cost and complexity.

We have initiated the extension of NRL's traditional SILM approach for characterizing or identifying the multi-field constitutive response of composites under mechanical and heat flux loading, to a DMM approach by appropriate modification of our mechatronic automation methodology [7]. Composite material characterization under thermal and mechanical loading is essential for the application area where DDEMA will be validated for the case of aircraft modeling activity. The appropriate design scenario involves mechanical pressure and heat fluxes that are applied on aircraft skins due to the structure-fluid interaction induced by supersonic flight conditions. The specific approach is based in extending the notion of dissipated energy density (DED). DED is the function that is traditionally constructed as a sum of basis functions weighted by 
unknown coefficients to be determined trough the identification process from mechatronically derived experimental data 7 . These coefficients represent the material model parameters and the procedure to identify them has always assumed isothermal and adiabatic conditions for the material under identification. However, the co-existence of aerodynamically induced heat fluxes has forced reconsideration of the isothermal and adiabatic assumptions for the identification process. There are multiple ways to account for a material that represents an open system that can exchange heat with its environment. Allowing temperature to be a state variable of the DED function of the system in addition to the strain components, is one of them. Another possible extension is to allow the parameters of the isothermal model to become functions of temperature themselves. Many other extensions can be constructed by considering varying combinations of both of the previous extensions. These extensions constitute the template domain for the DMM approach. A meta-optimization scheme outside each individual SLIM can select the best one of all these extensions and is the embodiment of the DMM methodology that will be further developed.

\section{Dynamic Data Driven Adaptive Simulation}

To enable and improve dynamic data driven adaptivity of systemic behavioral simulation with real-time performance, the analytical and computational complexity of the models involved requires that techniques and methodologies are developed and employed to accelerate computation. It is essential that incoming data originating from sensors associated with the actual physical systems are utilized to exercise their models in a manner that achieves this objective.

An extended description of the techniques available for reducing model complexity through reduced order modeling, and for reducing total computation time by implementing combined computational parallelism based in domain decomposition techniques in both space and time has been given elsewhere [4, 5, 6,

Here we are focusing on sensor-originating data-driven solution synthesis from pre-existing basis cases behavioral simulations.

Let $r_{i}$ represent a scalar field corresponding to a response component of the behavior of a continuous system indexed by the position index $i$ usually corresponding to some nodal identification address as it relates to a mesh used for determining it numerically. The context of this response component includes the existence of some analytical model (i.e. set of partial differential equations), some discretization scheme (i.e. using finite element/volume analysis over an appropriately selected mesh) that when applied yields the values of all output state variables of the system (i.e. temperature, strain components, concentrations etc.) as a column array $\mathbf{r}$ containing its values $r_{i}$ where $i=1, \ldots, m$ for all mesh points $m$. It is assumed that there is a column vector $\mathbf{f}$, of $n$ global input parameters $f_{j}, j=1, \ldots, n$, regarded as the forcing or loading inputs into our systemic simulation model in a manner that potentially effects all $r_{i}$ at every $i$ point. To obtain the base case solutions $\mathbf{b}_{j}$, we apply the solution scheme for our system's of PDE-based model $n$ times for each one of the forcing inputs by 
setting $f_{j}=1$ for each forcing function. We further postulate as in 8 , that there is an appropriate selection of forcing input parameters such as any response of the system can always be expressed as a linear combination of this base case solutions, according to,

$$
\mathbf{r}=\mathbf{B c},
$$

where $\mathbf{B}$ is the rectangular $n \times m$ array constructed such as $\mathbf{B}=\left[\mathbf{b}_{1}\left(f_{1}\right)\right.$, $\left.\cdots, \mathbf{b}_{n}\left(f_{n}\right)\right]$ that has elements defined by $B_{i j}=b_{i}\left(f_{j}\right)$, and $\mathbf{c}$ is an $n$-dimensional column array containing the proportion coefficients $c_{j}$ multiplying each one of the base cases to construct the final solution.

It is important to recognize that $\mathbf{B}$ can be computed prior to its utilization from Eq. 1 at the time of of solution synthesis for real-time simulation. In fact, the final behavioral solution synthesis is achieved by the computational implementation of Eq. 1 provided $\mathbf{c}$ is known.

Determination of $\mathbf{c}$ can be achieved via the use of $q$ sensors embedded in the physical system at positions $k$ that can be selected to coincide with some of the points $i$ and they measure the sensor response column vector $\mathbf{s}$ that contains individual sensor responses $s_{l}, l=1, \ldots, q$. Since $s_{l}$ essentially is $r_{l}$ at the point where a sensor exists, and without loss of generality we assume noiseless sensors, then we can repeat the previously applied argumentation and approximate the sensor response according to

$$
\mathbf{s}=\mathbf{Q} \mathbf{c}
$$

$\mathbf{Q}$ represents a limited version of $\mathbf{B}$ such that only the points on the mesh where sensors are located are considered. Thus $\mathbf{Q}$ is an $n \times q$ rectangular array. Clearly for any case where $n \leq q$ (i.e. base cases are less than the number of available sensors), there is a pseudo inverse $\mathbf{Q}^{+}$of $\mathbf{Q}$, such that Eq. 2 can be inverted to determine $\mathbf{c}$ and subsequently inserted in Eq. 10 to yield

$$
\mathbf{r}=\mathbf{B Q}^{+} \mathbf{s} \text {. }
$$

This relation allows to compose the response of the system in terms of precomputed and stored solutions (i.e. $\mathbf{B}$ and $\mathbf{Q}$ and their product) and sensor originating data (i.e. $\mathbf{s}$ ). In this manner, we avoid spending the time for reapplying the PDE solution scheme that involves time-consuming computational application of space and time integration (we only do this for the base cases), and substitute it to much more computationally efficient sequences of multiplications and additions as defined by the computational implementation of the matrix operations designated by Eq. 3. It is also worthwhile mentioning here that the solutions represented by $\mathbf{B}$ may be constructed in terms of reduced order models to further accelerate total computation time for both the pre-computing and real-time stages of the process.

Application 1: Aircraft Behavior Prediction. We have initiated work for applying the pre-computed synthesis approach described for the case of subsonic to supersonic regime of the AGARD wing 445.6 9. This wing is an AGARD standard aeroelastic configuration with a 45 degrees quarter-chord sweep angle, a panel aspect ratio of 1.65 , a taper ratio of 0.66 , and a NACA $65 \mathrm{~A} 004$ airfoil 
section. In our previous work [10 we have modeled and solved the three-field PDE system governing fluid-structure aeroelastic response of this wing and have established favorable comparisons with experimental results. Results for various state variables have been obtained for various flight conditions defined by an appropriate variation of the far-field mach number (denoting the speed of the corresponding aircraft) and angle of attack. These two parameters are also selected as the forcing input parameters for our system.

To apply the above mentioned scheme, we have selected the total strain energy density (SED) per unit of volume, as the behavioral response state variable of choice. In addition, we assumed the presence of 4 sensors that can measure in-plain strains on specific places of the wing. We focused on small region of the parameter space defined by $\left(M_{\infty} \in[0.6,0.8], \alpha \in\left[6^{\circ}, 8^{\circ}\right]\right)$, in order to allow proportional superposition as it is enforced by the composition equations.

Figure 1 shows nine SED distributions of the AGARD wing resulting for 3 values of $M_{\infty}$ and $\alpha$ respectively. All cases surrounded by dash-lined frames have been both synthesized and computed from the direct solution procedure. Differences between synthesized and direct SED distributions never exceeded 4.2 $\%$ root mean square error. The red dots shown in the case of $\left(M_{\infty}, \alpha\right)=\left(0.6,6^{\circ}\right)$ represent the location of the sensors.

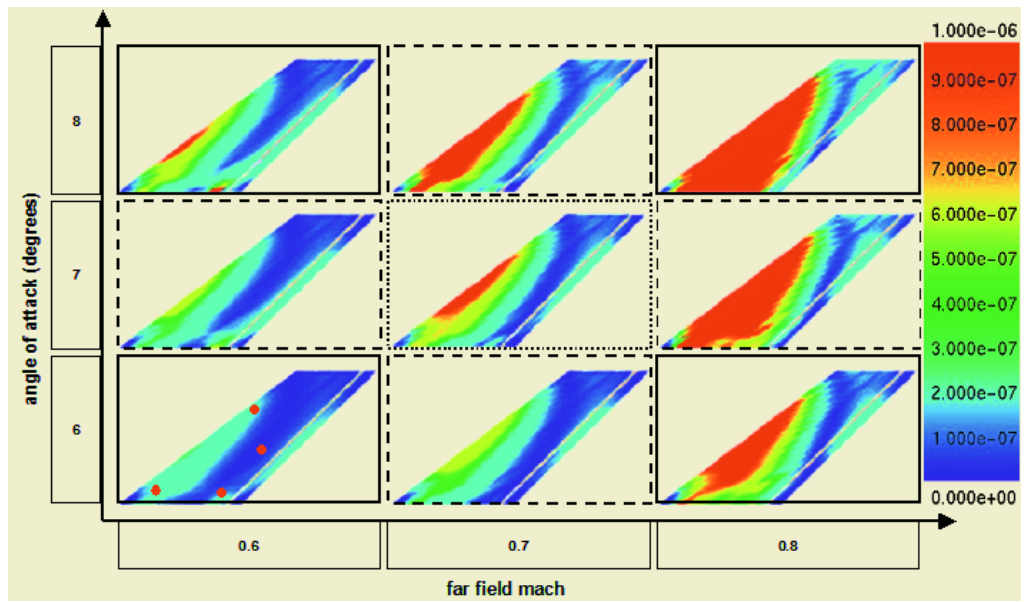

Fig. 1. Strain energy density distributions of the AGARD wing. All distributions along $M_{\infty}=0.7$ and $\alpha=7^{\circ}$ have been both computed and reconstructed and the differences are undiscernible

Direct computation of any of these solutions took an average of 19.6 minutes on six processors of a SGI Origin 2000. It is indicative that solution synthesis of the same case implemented as a GAWK script running on a $3.2 \mathrm{GHz}$ Pentium- 4 processor takes 0.6 seconds. Thus a realization of 1960 times speedup is achieved (without adjusting for the different processor unit number and architecture). 
Application 2: Fire Dynamics Behavior Prediction. Application of the solution synthesis approach based on dynamic sensor data requires the establishment the base case parameter space. The problem of fuel leak at room inside a submarine has been selected to represent the physical context for this case. "Fire Dynamic Simulator" (FDS) / citeFDS01 has been selected to numerically integrate the corresponding reactive flow PDE-model.

We have selected a rectangular $5 \times 3$ array of 15 thermocouple sensors that measure temperature. We have chosen the excitation parameters for constructing our base cases to be: total time combustion is ongoing ( $t_{c}$ in seconds); position-X of leak ( $f_{x}$ in meters); position-Y of leak ( $f_{y}$ in meters); fuel's Heat Release Rate Per Unit Area to designate the fire strength $\left(f_{s}\right.$ in $\left.\mathrm{kW} / \mathrm{m}^{2}\right)$. They define a four dimensional parameter space and in particular a bounded sub-region defined by $\left\{t_{c} \in[0 ., 10],. f_{x} \in[0 ., 4.7], f_{x} \in[0 ., 2.5], f_{s} \in[100 ., 500].\right\}$.

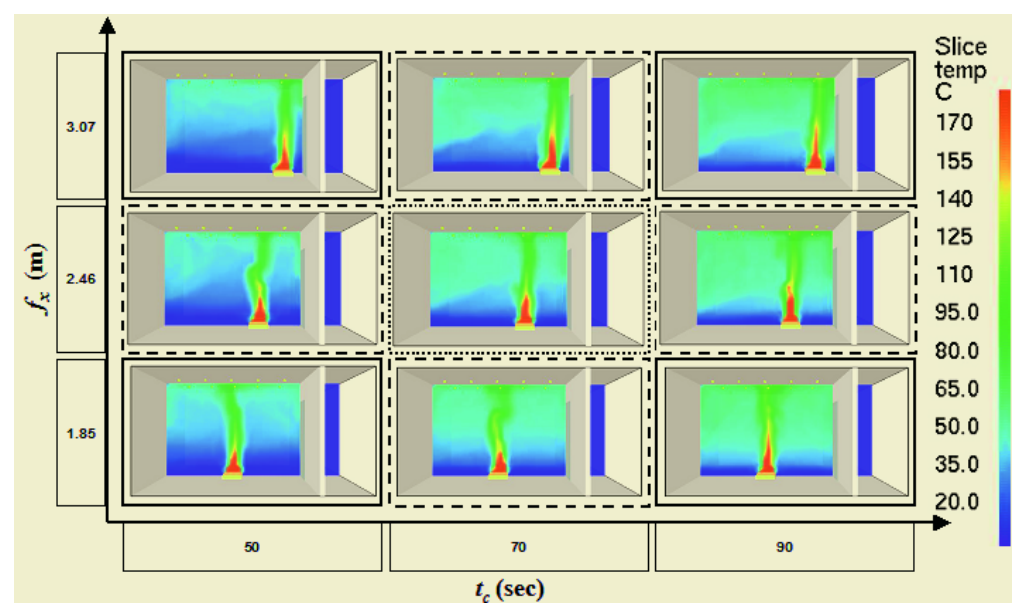

Fig. 2. Temperature distribution along $x$-axis at the mid section of a submarine room with a fuel leak. All the distributions for $t_{c}=70 \mathrm{sec}$ and $f_{x}=2.46 \mathrm{~m}$ have been both computed and reconstructed and the differences are undiscernible

While the average time for all FDS-computed cases was 13.4 minutes on a $3.2 \mathrm{GHz}$ Pentium 4 system, the synthesized solutions took 1.6 seconds on the same system. Therefore the average speedup factor was 817 . It should be noted that the synthesized runs in addition to the actual computation include the data input and output time.

\section{Computational Implementation Progress}

Progress regarding the computational implementation of DDEMA is reported for the cases that follow. 
Supersonic Platform Simulation: The core technology behind the aircraft multiphysics simulations for our project is the AERO suite of codes [12. To provide Operating System and system architecture transparency along with ease of use we have developed a JAVA-based front-end called JAERO. JAERO is a full JAVA stand-alone application that runs on the local client machine in front of the user and provides automated configuration, data integrity, dependency and consistency checking, file management, execution control and monitoring management services to the AERO suite installed on a remote cluster. JAERO does not enforce continuous master-slave relationship to the AERO suite executables and furthermore it provides job monitoring services at will. State storage and recovery has been enabled by utilization of XML-based file encoding, storage and recovery. It does not require HTTPD server presence since it exploits SSH client-server daemon based multi-threaded communications. RSA fingerprints are used for host authentication and encryption has been implemented for all data transmitted over the network. XML scripting of a full runtime session of AERO that encapsulates all the inter-code parametrization, data file location, cluster e.t.c is also provided and it will eventually provide extended availability to 3rd parties. JAERO is currently undergoing beta testing and is be extended to support data visualization post-processing.

Fire Dynamics Simulation: The core technology behind the reactive flow modeling and simulation for fire dynamics prediction applications, is the FDS legacy code. To enable lightweight parallelism capability on distributed heterogeneous networked systems, and Operating System and system architecture transparency along with ease of use, we have developed JAMPI. JAMPI is a set of resources for "J" ava "A" gent enabling of the "M" essage "P" assing "I"nterface for legacy MPI-based codes. In particular, we used JAMPI to develop the Java Fire Dynamic Simulator (JFDS) that resulted in converting the MPI version of FDS into a JADE / cite agent implementation of it. This approach enables instances of legacy codes installed on various shared memory architectures, to appear as light-weight agents accessing the codes directly or their corresponding GRID or WEB services encapsulation. Therefore, all benefits of using agentbased middleware are automatically realizable for legacy codes in general and for FDS in particular.

JAMPI is in alpha stage while JFDS is in beta testing stage and we are currently undergoing through validation procedures along with performance analysis and comparison studies.

\section{Acknowledgments}

The authors acknowledge the support by the National Science Foundation under grant ITR-0205663. Partial support from NRL's 6.1 core-funding is also greatly acknowledged. 


\section{References}

1. Michopoulos, J., Tsompanopoulou, P., Houstis, E., Rice, J., Farhat, C., Lesoinne, M., Lechenault, F., DDEMA: A Data Driven Environment for Multiphysics Applications,in: Proceedings of International Conference of Computational Science ICCS'03, Sloot, P.M.A., et al. (Eds.) Melbourne Australia, June 2-4, LNCS 2660, Part IV, Springer-Verlag, Haidelberg, (2003), 309-318.

2. Michopoulos, J., Tsompanopoulou, P., Houstis, E., Farhat, C., Lesoinne, M., Rice, J., Joshi, A., On a Data Driven Environment for Multiphysics Applications, Future Generation Computer Systems, in-print, (2005).

3. Michopoulos, J., Tsompanopoulou, P., Houstis, E., Joshi, A., Agent-based Simulation of Data-Driven Fire Propagation Dynamics, Proceedings, International Conference Computational Science - ICCS`04, Krakow, Poland, June 6-9, 2004, Part III Series: Lecture Notes in Computer Science, Bubak, M.; Albada, G.D.v.; Sloot, P.M.A.; Dongarra, J. (Eds.), LNCS 3038, (2004), 732-739.

4. Farhat, C., Chandesris, M., Time-Decomposed Parallel Time-Integrators: Theory and Feasibility Studies for Fluid, Structure, and Fluid-Structure Applications, Internat. J. Numer. Meths., Engrg. 58, (2003), 1397-1434.

5. Lieu, T., Lesoinne, M., Parameter Adaptation of Reduced Order Models for ThreeDimensional Flutter Analysis, $42^{\text {nd }}$ AIAA Aerospace Sciences Meeting and Exhibit, Reno, NV, (2004).

6. Michopoulos, J., Farhat, C., Houstis, E., Dynamic-Data-Driven Real-Time Computational Mechanics Environment, Proceedings, International Conference Computational Science - ICCS`04, Krakow, Poland, June 6-9, 2004, Part III Series: Lecture Notes in Computer Science, Bubak, M.; Albada, G.D.v.; Sloot, P.M.A.; Dongarra, J. (Eds.), LNCS 3038, (2004), 693-700.

7. Michopoulos, J.G., Mechatronically Automated Characterization of Material Constitutive Response, in Proceedings of the 6th World Congress on Computational Mechanics (WCCM-VI), September 5-10 2004, Beijing China, Tsinghua University Press and Springer, (2004), 486-491.

8. Michopoulos, J.G., Mast, P.W. , Badaliance, R., Wolock, I., Health Monitoring of smart structures by the use of dissipated energy, ASME proc. 93 WAM on Adaptive structures and material systems, G.P. Carman/E. Garcia, eds., ASME, AD-Vol. 35, (1993), 457-462.

9. Yates, E., AGARD Standard Aeroelastic Configuration for Dynamic Response, Candidate Configuration I. - Wing 445.6, NASA TM-100492, (1987).

10. Michopoulos,J. , Badaliance, R. , Chwastyk, T. , Gause, L., Mast, P., Farhat C. , and Lesoinne M., Material Softening Issues In A Multiphysics Virtual Wind Tunnel Environment, AIAA 2002-1095, proceedings of the 2002 AIAA conference at Reno Nevada January 14-17 2, (2002).

11. McGrattan, K.B., Baum, H.R., Rehm, R.G., Hamins, A., Forney, G.P., Fire Dynamics Simulator - Technical Reference Guide, National Institute of Standards and Technology, Gaithersburg, MD., NISTIR 6467, (2000).

12. Farhat, C., Geuzaine, P., Brown, G., Application of a Three-Field Nonlinear FluidStructure Formulation to the Prediction of the Aeroelastic Parameters of an F-16 Fighter, Computers and Fluids, Vol. 32, (2003), 3-29. 